\title{
Crystal structure of AcrB complexed with linezolid at $3.5 \AA$ resolution
}

\author{
Li-Wei Hung $\cdot$ Heung-Bok Kim · Satoshi Murakami • \\ Goutam Gupta $\cdot$ Chang-Yub Kim $\cdot$ Thomas C. Terwilliger
}

Received: 17 April 2013/Accepted: 4 May 2013/Published online: 15 May 2013

(C) The Author(s) 2013. This article is published with open access at Springerlink.com

\begin{abstract}
AcrB is an inner membrane resistance-nodulation-cell division efflux pump and is part of the AcrABTolC tripartite efflux system. We have determined the crystal structure of AcrB with bound Linezolid at a resolution of $3.5 \AA$. The structure shows that Linezolid binds to the A385/F386 loops of the symmetric trimer of AcrB. A conformational change of a loop in the bottom of the periplasmic cleft is also observed.
\end{abstract}

Keywords Multidrug resistance $\cdot$ AcrB $\cdot$ RND efflux pumps · Linezolid · Membrane protein · Protein-drug complex $\cdot \mathrm{X}$-ray crystal structure

$\begin{array}{ll}\text { Abbreviations } \\ \text { AcrA } & \text { Acriflavine resistance protein A } \\ \text { AcrB } & \text { Acriflavine resistance protein B } \\ \text { ASU } & \text { Asymmetric unit } \\ \text { FDA } & \text { US food and drug administration } \\ \text { Linezolid } & \begin{array}{l}(S)-N-(\{3-[3-\text { fluoro-4-(morpholin-4-yl) } \\ \text { phenyl]-2-oxo-1,3-oxazolidin-5-yl }\}\end{array} \\ & \begin{array}{l}\text { methyl)acetamide } \\ \text { MIC }\end{array} \\ \text { Minimal inhibitory concentration } \\ \text { MDR } & \text { Multidrug resistance }\end{array}$

L.-W. Hung $(\bowtie) \cdot$ H.-B. Kim · G. Gupta $\cdot$ C.-Y. Kim ·

T. C. Terwilliger (西)

Los Alamos National Laboratory, Los Alamos, NM 87545, USA

e-mail: 1whung@lanl.gov

T. C. Terwilliger

e-mail: terwilliger@lanl.gov

S. Murakami

Life Science Department, School and Graduate School

of Bioscience and Biotechnology, Tokyo Institute of

Technology, J2-17, 4259 Nagatsuta-cho, Midori-ku,

Yokohama 226-8503, Japan
NMP
PEG
PDB
RND
TM
1-(1-Naphthylmethyl)-piperazine
Polyethylene glycol
Protein data bank
Resistance nodulation division
Transmembrane

\section{Introduction}

AcrB, the principal multidrug transporter in E. coli, crosses the cytoplasmic membrane and acts as a proton/drug antiporter. It is part of the AcrAB-TolC tripartite efflux system consisting of an outer membrane factor, TolC, a periplasmic membrane fusion protein, AcrA, and the inner membrane resistance-nodulation-cell division (RND) efflux pump, AcrB. This integrated three-component molecular complex extrudes a large variety of cytotoxic substances such as antibiotics, organic solvents, dyes, and detergents from the cell directly into the medium, bypassing the periplasm and the outer membrane [1-3]. Over-expression of the tripartite RND efflux systems is thought to be a major factor in multidrug resistance (MDR) in Gram-negative bacteria. Effective control of this and related MDR systems has become an emerging focus for global public health efforts $[2,4]$.

AcrB is one of the most well-studied RND efflux pumps. Crystal structures of AcrB by itself as well as several drugbound complexes have been structurally characterized [5-12]. Two types of quaternary arrangement have been observed in structures of AcrB: (1) a symmetric trimer, formed by three identical protomers or protomer-drug complexes; and (2) an asymmetric trimer, consisting of three distinct protomer conformations corresponding to three functional states of the transport cycle: access, binding and extrusion, with drug molecules only bound to 
the binding protomer. These structures have exemplified several drug-binding modes of AcrB and provided structural insights to the substrate transport mechanism of RND multidrug efflux transporters.

In an effort to explore how AcrB interacts with drugs, we have determined the crystal structure of AcrB complexed with Linezolid, an oxazolidinone-type antibacterial agent that inhibits bacterial protein synthesis by specifically binding to the 50S ribosomal subunit. Linezolid was the first FDA-approved oxazolidinone antibiotic used for the treatment of serious infections caused by Gram-positive bacteria that are resistant to other antibiotics. Therefore, this drug has been called a "reserve antibiotic", a drug of last resort against potentially intractable infections [13]. Linezolid is a synthetic compound, and is therefore not susceptible to the same mechanisms underlying bacterial resistance against naturally occurring antibiotics. However, it has no clinically significant effect on most Gram-negative bacteria. This is thought to be a result of relatively low intracellular concentration of Linezolid due to efflux [14]. The intracellular concentration of Linezolid could be increased substantially by inhibition of RND-type efflux pumps [14]. E. coli with inactivated AcrB has been found to be more susceptible to Linezolid than cells with an intact pump [15]. Further, NMP (1-(1-naphthylmethyl)-piperazine, a putative efflux pump inhibitor) has been shown to reduce the MIC (minimal inhibitory concentration) of Linezolid by fourfold for E. coli, Citrobacter freundii, Enterobacter aerogenes and Acinetobacter baumannii [16, 17]. Although these data suggest that Linezolid can be extruded by efflux pumps, there is no direct evidence yet to support this hypothesis. Here, we report the crystal structure of AcrB and Linezolid complex, in which AcrB indeed binds Linezolid in the same fashion as several other antibiotics that are extruded by efflux pumps.

\section{Materials and methods}

Cloning, overexpression, and purification

Wild-type AcrB with a C-terminal polyhistidine tag was prepared as described previously [7]. Briefly, AcrB was overproduced in E. coli JM109 with a histidine-tagged AcrB-overexpersion plasmid pAcBH. The cells were disrupted with Microfluidizer (Microfluidics Corp.) and the membrane fractions were collected washed using several ultracentrifugation steps at $150,000 \mathrm{~g}$ for $90 \mathrm{~min}$. Purified membranes were solubilized in buffer containing $50 \mathrm{mM}$ Tris- $\mathrm{HCl}, \mathrm{pH} 7.0,10 \%$ glycerol in $2 \% n$-dodecyl- $\beta$-Dmaltoside (DDM) (Anatrace). Lipids and debris were removed by ultracentrifugation at $170,000 \mathrm{~g}$ for $60 \mathrm{~min}$. Extracted histidine-tagged AcrB was purified with metal affinity column chromatography equilibrated with buffer (20 mM Tris-HCl, pH 7.5, 0.3 M NaCl, $10 \%$ glycerol and $0.2 \%$ DDM). The column was washed stepwise using 25 and $100 \mathrm{mM}$ imidazole added to the above buffer. Purified AcrB was eluted with $300 \mathrm{mM}$ imidazole. Imidazole was then removed by three concentration-dilution steps using an ultrafiltration membrane. Proteins were concentrated to $28 \mathrm{mg} / \mathrm{mL}$ in $20 \mathrm{mM}$ sodium phosphate (pH 6.2), $10 \%$ (v/v) glycerol and $0.2 \%$ (w/v) DDM and were frozen in liquid nitrogen.

\section{Crystallization and data collection}

AcrB was crystallized using the sitting-drop vapor diffusion method with $0.1 \mathrm{M} \mathrm{NaCl}$, Na-phosphate $\mathrm{pH} 6.2$, and $8 \%$ PEG 4000 as crystallization reagents. Crystals of the AcrBLinezolid complex were obtained by soaking the AcrB crystals in a solution containing Linezolid prior to data collection. Linezolid stock solution $(30 \mathrm{mM})$ was prepared with water, and $6 \mathrm{mM}$ Linezolid was added to a cryosolvent containing the crystallization reagents plus $25 \%$ glycerol. Apo-AcrB crystals were transferred to the cryosolvent and incubated at $21{ }^{\circ} \mathrm{C}$ for $10 \mathrm{~min}$ before flash cooling in liquid nitrogen. X-ray diffraction data were collected at $100 \mathrm{~K}$ on the 5.0.2 beam line at the Advanced Light Source at the Lawrence Berkeley National Laboratory with X-rays at a wavelength of $1 \AA$. The crystal diffracted better than $3.3 \AA$ initially and decayed during data collection, leading to a useful resolution of about $3.5 \AA$ by the end of data collection. The diffraction data were processed with the HKL2000 program suite [18]. The AcrB-Linezolid complex belongs to the space group $R 32$ with cell parameters $a=b=$ $144.7 \AA, c=519.4 \AA$. The solvent content is $74 \%$ assuming 1 molecule in the asymmetric unit.

Structure determination and refinement

The structure determination and refinement was conducted with the Phenix program suite [19]. The apo-AcrB crystal structure (1IWG, [7]), obtained using conditions similar to those used here and having the same space group and very similar cell parameters $(a=b=144.5 \AA$, $c=519.2 \AA$ ) was used as starting model for refinement using phenix.refine [20]. The asymmetric unit of the crystal contains one chain of AcrB. One round of rigid-body refinement followed by B-factor refinement yielded an $\mathrm{R}$-factor of $30.9 \%$. The refinement was continued with several cycles of positional, B-factor, and TLS refinement, and revealed clear difference density (Fig. 1a, green, contoured at $3 \sigma$ ) near residue F386. Increasing the contour level of the Fo-Fc map to $3.6 \sigma$ revealed 3 separate blobs (Fig. 1a, magenta) suggesting that the density consisted of 3 electron-dense substructures, consistent with the three 
separate electron-dense parts of Linezolid. A Linezolid was therefore modelled into this density. In addition, residues 860-864 were rebuilt into the electron density map as they were not modelled in the starting model. The structure was manually inspected and minor changes were made in Coot [21], followed by refinement until convergence. The final refined structure has an R-factor of $25.1 \%$ and a free-R factor of $30.4 \%$ for data between 50 and $3.5 \AA$. These are similar to the R-factors for the earlier AcrB structure (29.0/ $35.5 \%)$. As a comparison, the R- and free R-factors of AcrB-Linezolid complex without TLS refinement were 29.8 and $33.7 \%$, respectively. Detailed statistics are shown in Table 1. The final refined Linezolid model and density are shown in Fig. $1 b$.

\section{Structure validation and deposit}

The quality of the final structure was assessed using MolProbity [22] and phenix.refine. The atomic coordinates and structure factors are available in the Protein Data Bank under accession code 4K7Q.

\section{Results and discussion}

Overall structure of AcrB-linezolid complex

Figure 2 shows a ribbon diagram of the complex structure of AcrB-Linezolid with Linezolid molecules shown in space-filling models. The AcrB crystal used for Linezolid soaking was in the $R 32$ space group in which symmetric trimers form in the unit cell. Each AcrB monomer contains a transmembrane (TM) domain consisting of 12 TM helices, and two periplasmic domains, the porter domain, and the TolC-binding domain [7]. The AcrB monomers form a trimer which appears to be stabilized by the inter-monomer locking loops protruding into the adjacent AcrB monomer in the TolC binding domain. The interlocked TolC-binding domains form a funnel-like structure at the top and a connected tunnel at the center. The tunnel leads through the porter domain down to the large central cavity formed by the TM domains of the three protomers. The central cavity is connected also to the periplasm through three vestibules located at subunit interfaces. These vestibules have been shown to play important roles in substrate capture and transport [23]. A cleft at the periplasmic periphery of the porter domain has been suggested to accommodate AcrA [7], and has been shown to be crucial for substrate binding and transport of AcrB [24, 25].

Each protomer of the symmetric AcrB trimer binds one Linezolid molecule on the wall of the upper portion of the central cavity near residues A385 and F386. The 3 rings of the Linezolid molecule lie approximately parallel to the F386 binding loop, allowing maximum hydrophobic contact between the drug and AcrB (Fig. 3). The binding interface buries approximately $140 \AA^{2}$ surface of Linezolid (calculated with areaimol, [26]). Almost the whole Linezolid except for its acetamide tail participates in this intermolecular interaction dominated by hydrophobic stacking. Several other drugs including ethidium [12], nafcillin [11] and ampicillin [10] also have been found to bind to this location on the symmetric AcrB trimers.

\section{Comparison with apo-AcrB structure}

The overall structure of AcrB-Linezolid complex was very similar to that of AcrB by itself [7]. There was a significant local conformational difference at residues 670-675. When superposed together, the two structures has a root-meansquare C $\alpha$ coordinate deviation of $0.4 \AA$ while these residues, on the surface of the protein but not near crystal contacts, differ by up to $4 \AA$ in backbone position. These residues reside in a loop lining the bottom of the cleft in the porter domain thought to be important for substrate transport and AcrA binding. It is possible that the change in position of this loop between unbound and Linezolid-
Fig. 1 a Unbiased Fo-Fc difference Fourier map contoured at $3 \sigma$ (green) and $3.6 \sigma$ (magenta) with a Linezolid molecule overlaid onto the density. No Linezolid had been modeled and refined at this stage. b Final refined $2 \mathrm{Fo}-\mathrm{Fc}$ map contoured at $1 \sigma$ (a)

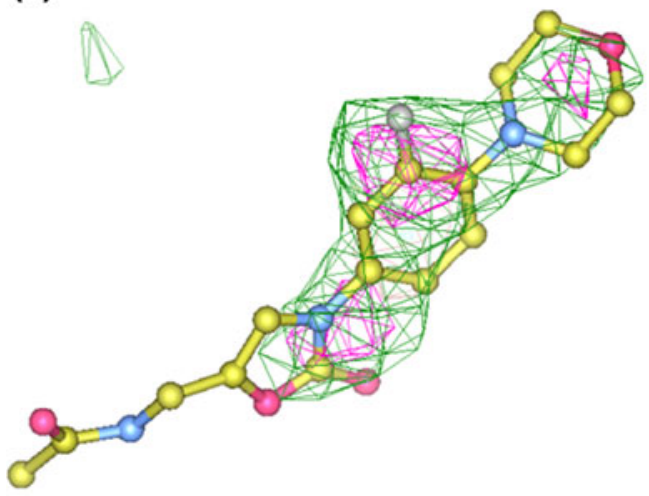

(b)

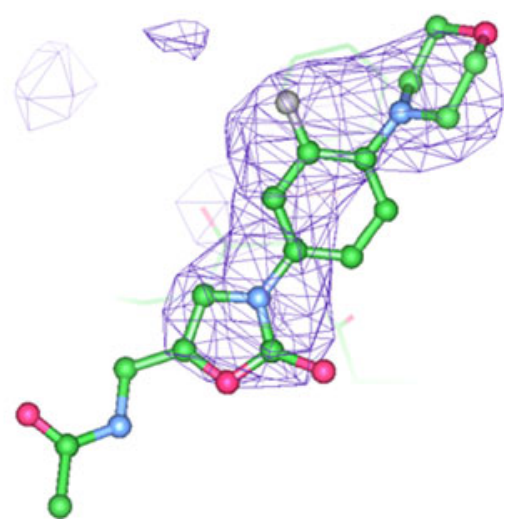


Table 1 X-ray data and refinement statistics

\begin{tabular}{|c|c|}
\hline \multicolumn{2}{|l|}{ Crystal parameters } \\
\hline Space group & R 32 \\
\hline \multicolumn{2}{|l|}{ Cell dimensions } \\
\hline $\mathrm{a}, \mathrm{b}, \mathrm{c}(\AA)$ & $144.7,144.7,519.4$ \\
\hline$\alpha, \beta, \gamma\left({ }^{\circ}\right)$ & $90,90,120$ \\
\hline Matthews coefficient $\left(\AA^{3} / \mathrm{Da}\right)$ & 4.8 \\
\hline Solvent content $(\%)$ & 74.2 \\
\hline \multicolumn{2}{|l|}{ Data collection $^{\mathrm{a}}$} \\
\hline Wavelength $(\AA)$ & 1.0 \\
\hline Resolution $(\AA)$ & $46.6-3.5(3.56-3.50)$ \\
\hline $\mathrm{R}_{\text {merge }}(\%)$ & $6.1(78.8)$ \\
\hline No. of unique reflections & 25,688 \\
\hline No. of reflections in $R_{\text {free }}$ set & 1,788 \\
\hline Mean redundancy & $5.8(5.9)$ \\
\hline Overall completeness $(\%)$ & $99.8(100.0)$ \\
\hline Mean $I / \sigma$ & $28.0(2.2)$ \\
\hline \multicolumn{2}{|l|}{ Refinement residuals ${ }^{\mathrm{b}}$} \\
\hline $\mathrm{R}_{\text {free }}(\%)$ & $30.36(44.0)$ \\
\hline $\mathrm{R}_{\mathrm{work}}(\%)$ & $25.13(35.9)$ \\
\hline Completeness (\%) & $95.3(56.1)$ \\
\hline \multicolumn{2}{|l|}{ Model quality } \\
\hline RMSD bond lengths $(\AA)$ & 0.003 \\
\hline RMSD bond angles $\left(^{\circ}\right)$ & 0.767 \\
\hline \multicolumn{2}{|l|}{$\begin{array}{l}\text { MolProbity Ramachandran } \\
\text { distribution }\end{array}$} \\
\hline Most favored (\%) & 88.0 \\
\hline Allowed (\%) & 9.7 \\
\hline Disallowed (\%) & 2.3 \\
\hline Mean main chain B-factor $\left(\AA^{2}\right)$ & 130 \\
\hline Mean overall B-factor $\left(\AA^{2}\right)$ & 133 \\
\hline Mean solvent B-factor $\left(\AA^{2}\right)$ & N/A \\
\hline \multicolumn{2}{|l|}{ Model contents } \\
\hline Protomers in ASU & 1 \\
\hline Protein residues & $\begin{array}{l}\text { A }(7-498,513-864 \\
869-1,036)\end{array}$ \\
\hline Ligands & 1 Linezolid \\
\hline No. of protein atoms & 7,676 \\
\hline No. of ligand atoms & 24 \\
\hline No. of water molecules & 0 \\
\hline PDB accession code & 4K7Q \\
\hline
\end{tabular}

Standard definitions were used for all parameters. Entries in parentheses report data from the limiting resolution shell. Data collection and refinement statistics come from HKL2OOO and PHENIX, respectively. The abbreviations RMSD and ASU stand for root-mean-square deviation and asymmetric unit, respectively

a All observations with $\mathrm{I} \geq-3 \sigma_{\mathrm{I}}$ were included in calculating dataquality statistics. Rmerge $=\Sigma$ hkl $\Sigma \mathrm{i}|\mathrm{Ii}(\mathrm{hkl})-<\mathrm{I}(\mathrm{hkl})>| / \Sigma \mathrm{hkl} \Sigma \mathrm{i}$ Ii(hkl) where $\mathrm{Ii}(\mathrm{hkl})$ is the intensity of the ith observation and $<\mathrm{I}(\mathrm{hkl})>$ is the mean intensity of the reflections

b The crystallographic R factor $\mathrm{R}=\Sigma$ hkl $\mid$ |Fobs $|-|$ Fcall $\mid / \Sigma$ hkl $\mid$ Fobsl; Rfree $=\Sigma$ hkl || Fobs $|-|$ Fcall $\mid / \Sigma$ hkl $\mid$ Fobs $\mid$ where all reflections belong to a test set of randomly selected data

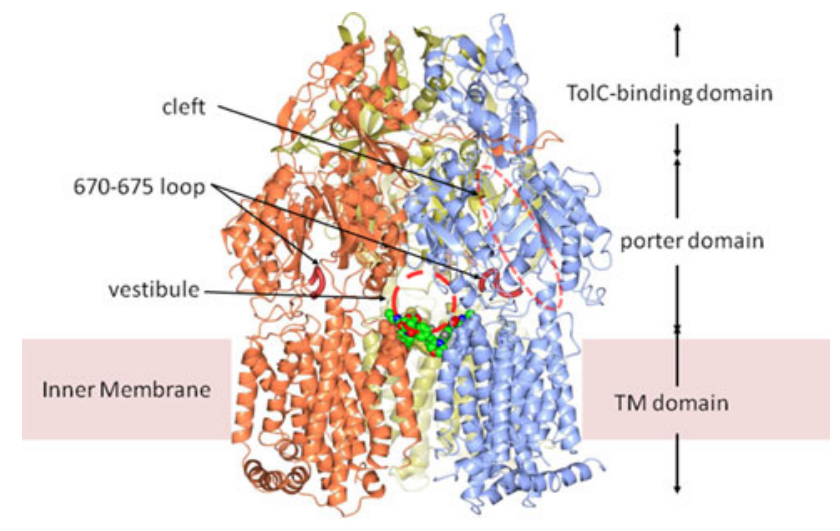

Fig. 2 Schematic diagram of AcrB-Linezolid complex. The Linezolid molecules are in colored space-filling model, viewable through one of the vestibule tunnels. The symmetric AcrB protomers are in ice-blue, coral, and gold ribbons. The 670-675 loops are shown in crimson tubes as depicted. Other components of the AcrB trimer described in the text are also labelled. This figure was created with CCP4 mg [27]

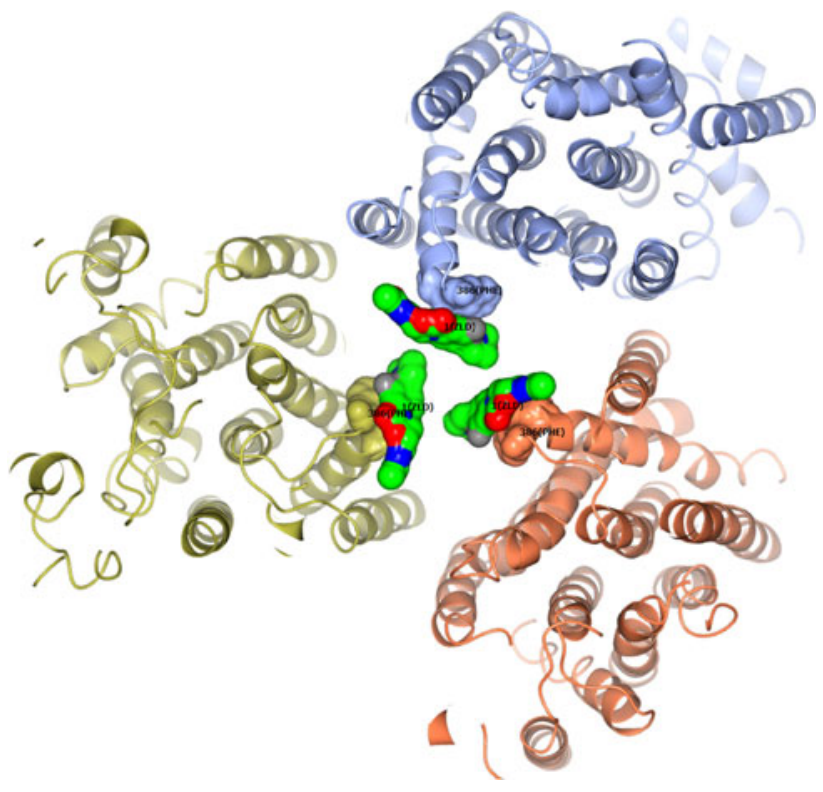

Fig. 3 Molecular contacts of AcrB trimer and Linezolid. The view is centered on the threefold axis on the periplasmic side looking down the inner membrane. The helixes shown are TMs of AcrB. Residues A385 and F386 of AcrB as well as Linezolid molecules are shown in colored surface models. Positions of F386 and Linezolid (ZLD) are labeled. The rest of the AcrB are in ribbons representation. The Linezolid molecules are in green, blue, red, colors indicating positions of $\mathrm{C}, \mathrm{N}, \mathrm{O}$ atoms, respectively. The colors are not indications of charges or potentials. This figure was prepared with CCP4 mg

bound states may reflect a functionally important state of AcrB, though we cannot rule out the possibility that the shift in the 670-675 loop was due to slight differences between crystals used for structure determination. We are in the process of obtaining higher resolution data of the 
AcrB-Linezolid complex as well as AcrB by itself under identical crystallization and data collection conditions.

\section{Summary}

The crystal structure of an AcrB-Linezolid complex has been determined at a resolution of $3.5 \AA$. The structure shows that one Linezolid binds to the A385/F386 loop of each protomer in the symmetric trimer. This loop has previously been shown to interact with Ethidium, Nafcillin, and Ampicillin. A conformational change is also found in a loop at the bottom of the periplasmic cleft thought to be important for AcrA binding and drug transport.

Acknowledgments The authors thank the Los Alamos National Laboratory Directed Research and Development Program for a Feasibility Studies Program for Study grant for support of this work. This work is partially supported by the funding program for Next Generation World-Leading Researchers (NEXT Program) and the program for Promotion of Fundamental Studies in Health Sciences of the National Institute of Biomedical Innovation (NIBIO) and by the ERATO "Lipid Active Structure Project" from Japan Science and Technology Agency (JST). We would like to thank the staff at the beam lines 5.0.1 and 5.0.2 managed by the Berkeley Center for Structural Biology (BCSB) at the Advanced Light Source (ALS) for technical support. The BCSB is supported in part by the National Institutes of Health, National Institute of General Medical Sciences, and the Howard Hughes Medical Institute. The ALS is supported by the Director, Office of Science, Office of Basic Energy Sciences, of the U.S. Department of Energy under Contract No. DE-AC0205CH11231.

Open Access This article is distributed under the terms of the Creative Commons Attribution License which permits any use, distribution, and reproduction in any medium, provided the original author(s) and the source are credited.

\section{References}

1. Nikaido H, Takatsuka Y (2009) Biochim Biophys Acta 1794(5):769-781

2. Seeger MA, Schiefner A, Eicher T, Verrey F, Diederichs K, Pos KM (2006) Science 313(5791):1295-1298

3. Ankarloo J, Wikman S, Nicholls IA (2010) Int J Mol Sci 11(4):1403-1412

4. Perez F, Hujer AM, Hujer KM, Decker BK, Rather PN, Bonomo RA (2007) Antimicrob Agents Chemother 51(10):3471-3484
5. Eicher T, Cha HJ, Seeger MA, Brandstatter L, El-Delik J, Bohnert JA, Kern WV, Verrey F, Grutter MG, Diederichs K, Pos KM (2012) Proc Natl Acad Sci USA 109(15):5687-5692

6. Murakami S, Nakashima R, Yamashita E, Matsumoto T, Yamaguchi A (2006) Nature 443(7108):173-179

7. Murakami S, Nakashima R, Yamashita E, Yamaguchi A (2002) Nature 419(6907):587-593

8. Nakashima R, Sakurai K, Yamasaki S, Nishino K, Yamaguchi A (2011) Nature 480(7378):565-569

9. Sennhauser G, Amstutz P, Briand C, Storchenegger O, Grutter MG (2007) PLoS Biol 5(1):e7

10. Tornroth-Horsefield S, Gourdon P, Horsefield R, Brive L, Yamamoto N, Mori H, Snijder A, Neutze R (2007) Structure 15(12):1663-1673

11. Yu EW, Aires JR, McDermott G, Nikaido H (2005) J Bacteriol 187(19):6804-6815

12. Yu EW, McDermott G, Zgurskaya HI, Nikaido H, Koshland DE Jr (2003) Science 300(5621):976-980

13. Wilson AP, Cepeda JA, Hayman S, Whitehouse T, Singer M, Bellingan G (2006) J Antimicrob Chemother 58(2):470-473

14. Schumacher A, Trittler R, Bohnert JA, Kummerer K, Pages JM, Kern WV (2007) J Antimicrob Chemother 59(6):1261-1264

15. Bohnert JA, Kern WV (2005) Antimicrob Agents Chemother 49(2):849-852

16. Kern WV, Steinke P, Schumacher A, Schuster S, von Baum H, Bohnert JA (2006) J Antimicrob Chemother 57(2):339-343

17. Schumacher A, Steinke P, Bohnert JA, Akova M, Jonas D, Kern WV (2006) J Antimicrob Chemother 57(2):344-348

18. Otwinowski Z, Minor W (1997) Processing of X-ray Diffraction Data Collected in Oscillation Mode. In: Carter CWJ, Sweet RM (eds) Methods in Enzymology, 276th edn. Academic Press, New York, pp 307-326

19. Adams PD, Afonine PV, Bunkoczi G, Chen VB, Davis IW, Echols N, Headd JJ, Hung LW, Kapral GJ, Grosse-Kunstleve RW, McCoy AJ, Moriarty NW, Oeffner R, Read RJ, Richardson DC, Richardson JS, Terwilliger TC, Zwart PH (2010) Acta Crystallogr D Biol Crystallogr 66(Pt 2):213-221

20. Afonine PV, Grosse-Kunstleve RW, Echols N, Headd JJ, Moriarty NW, Mustyakimov M, Terwilliger TC, Urzhumtsev A, Zwart PH, Adams PD (2012) Acta Crystallogr D Biol Crystallogr 68(Pt 4):352-367

21. Emsley P, Lohkamp B, Scott WG, Cowtan K (2010) Acta Crystallogr D Biol Crystallogr 66(Pt 4):486-501

22. Chen VB, Arendall WB 3rd, Headd JJ, Keedy DA, Immormino RM, Kapral GJ, Murray LW, Richardson JS, Richardson DC (2010) Acta Crystallogr D Biol Crystallogr 66(Pt 1):12-21

23. Husain F, Bikhchandani M, Nikaido H (2011) J Bacteriol 193(20):5847-5849

24. Husain F, Nikaido H (2010) Mol Microbiol 78(2):320-330

25. Takatsuka Y, Nikaido H (2010) Methods Mol Biol 634:343-354

26. Saff EB, Kuijlaars ABJ (1997) Math Intell 19:5-11

27. McNicholas S, Potterton E, Wilson KS, Noble ME (2011) Acta Crystallogr D Biol Crystallogr 67(Pt 4):386-394 